\title{
JEAN-CLAUDE PINSON : UNE « POÉTHIQUE » DU « POÉTARIAT »
}

\author{
Irati Fernández Erquicia \\ Universidad del País Vasco / Euskal Herriko Unibertsitatea \\ fernandezirati@gmail.com
}

RÉSUMÉ: Poète et philosophe contemporain, Jean-Claude Pinson explore volontiers sa double démarche philosophique et poétique, qui est précisément celle qui octroie à son travail avec les mots sa spécificité.

Dans cet article, nous étudierons deux des notions les plus évoquées par Pinson: celle de «poéthique», avec laquelle il tente d'explorer la part de vécu que la poésie s'efforce de saisir, et celle de «poétariat», qui désigne l'être capable de résister à l'émergence du capitalisme culturel et au grand développement des technologies de la communication. Pour l'étude de ces notions, nous aurons recours à plusieurs essais théoriques et philosophiques de Pinson, ainsi qu'à des extraits de certains de ses recueils poétiques, qui nous permettront de conclure qu'il souhaite maintenir un rapport permanent entre poésie et philosophie pour ainsi s'emparer de la dimension existentielle au sein du poème, et parvenir de cette manière à refonder un lien plus tangible avec le réel et les individus dans un contexte contemporain où nous tendons vers la perte de l'expérience du réel.

MOTS CLÉS: Jean-Claude Pinson, poéthique, poétariat, poésie contemporaine, philosophie, vécu.

\section{JEAN-CLAUDE PINSON: UNA "POÉTICA” DEL “POETARIADO”}

RESUMEN: Poeta y filósofo contemporáneo, Jean-Claude Pinson explora a menudo su doble enfoque filosófico y poético, que es precisamente el que otorga su especificidad a su trabajo con las palabras.

En este artículo estudiaremos dos de las nociones más evocadas por Pinson, la de la "poética" (poesía y ética), con la que intenta explorar la parte de existencia que la poesía trata de captar, y la de "poetariado", que describe al ser capaz de resistir al surgimiento del capitalismo cultural y al gran desarrollo de las tecnologías de la comunicación. Para estudiar dichas nociones, recurriremos a diferentes ensayos filosóficos y teóricos de Pinson, así como a algunos fragmentos 
de sus poemarios, que nos permitirán concluir que su trabajo desea mantener una relación permanente entre la poesía y la filosofía para así captar la dimensión existencial en el poema, y conseguir de esa manera refundar un vínculo más tangible con la realidad y con los individuos, en un contexto contemporáneo en el que tenemos tendencia a la pérdida de experiencia con la realidad.

PALABRAS CLAVE: Jean-Claude Pinson, poética, poetariado, poesía contemporánea, filosofía, existencia.

\title{
JEAN-CLAUDE PINSON: A "POÉTHIQUE" OF THE "POÉTARIAT"
}

\begin{abstract}
Contemporary poet and philosopher, Jean-Claude Pinson explores often his double philosophic and poetic approach, that makes the specificity of his work with words.

In this article, we will study two of the most developed notions by Pinson: the "poéthique", that tries to explore the part of existence that poetry attempts to capture, and the "poétariat", that describes the human being been able to resist to the emergence of cultural capitalism and the great development of new technologies of communication. To study those notions, we will turn to different essays and theorical works of Pinson, and to some poetic collections that will give us the opportunity to conclude that his work wants to maintain a permanent connection between poetry and philosophy, to capture an existential dimension on poems that will re-establish a more tangible connection with reality and with individuals, in a contemporary context in which we tend to a loss of experience with reality.

KEYWORDS: Jean-Claude Pinson, poéthique, poétariat, contemporary poetry, philosophy, existence.
\end{abstract}

Recibido: 16/11/2018. Aceptado: 17/01/2019

\section{Introduction}

Né en 1947 près de Nantes, Jean-Claude Pinson est un poète et philosophe qui semble toujours se débattre entre la philosophie et la poésie, un binôme qu'il accepte volontiers et auquel il n'est pas prêt à renoncer. D'ailleurs, c'est précisément ce partage permanent qui le caractérise sans doute le mieux, ainsi qu'il l'affirme lui-même dans son site officiel : 


\begin{abstract}
« Philosophe et poète » ? On m’accole souvent cette étiquette. Que je préfère barrée, tant elle me semble pompeuse et emphatique.

Je ne peux nier néanmoins que les livres que j’écris se partagent entre philosophie et poésie (quand ils ne mêlent pas les deux).

J'assume par contre pleinement la conjonction (« philosophe et poète »). Je n’ai jamais voulu choisir, ne voulant renoncer ni au est de l'ontologie ni au il y a de la poésie (Pinson, site officiel).
\end{abstract}

Ce partage permanent entre philosophie et poésie permet à Pinson d'explorer les limites qui séparent la philosophie et la poésie ou, plutôt, de parcourir les espaces que ces deux disciplines partagent, ainsi que nous pouvons le constater dans son essai Poéthique : une autothéorie (2013), où Pinson emploie le terme « poésophie » pour décrire sa conception poétique, qu'il envisage toujours aux côtés de la philosophie : «C'est bien sous l'angle de la philosophie que j’envisage en effet la poésie » (2013: 35).

Avec des recueils poétiques comme Laïus au bord de l'eau (1993), Fado (avec flocons et fantômes) (2001), Free Jazz (2004), Drapeau rouge (2008b), ou Alphabet cyrillique (2016), et des essais théorico-critiques comme Habiter en poète : Essais sur la poésie contemporaine (1995), À quoi bon la poésie aujourd'hui ? (1999), Sentimentale et naïve : nouveaux essais sur la poésie contemporaine (2002), À Piatigorsk, sur la poésie (2008a), ou Poéthique : une autothéorie (2013) entre autres, Pinson a inlassablement repris la question de la continuité, la survie et l'existence même de la poésie de nos jours : « Dans mes essais, je n'ai cessé de revenir sur la question du pourquoi, aujourd'hui, de la poésie. Pourquoi encore la désirer quand le monde tel qu'il va semble lui dénier toute importance ? » (Pinson, site officiel). Pinson tente de répondre à cette question du devenir de la poésie aussi bien dans ses travaux poétiques que dans ses essais philosophiques ou théoriques : en tant que poète, il met en place une poésie qui souhaite dire le vécu sur la page et, en tant que philosophe, il étudie la place consacrée à la poésie dans notre société contemporaine post-industrielle, marquée par ce que Pinson lui-même qualifie de « capitalisme culturel » (Pinson 2012), en faisant référence au contrôle social exercé par la médiatisation et la communication instantanée.

Pour situer son travail poétique, il nous faut parcourir brièvement les principales étapes poétiques qui l’ont précédé et qui nous permettront de lui attribuer une place au sein du panorama poétique actuel. Ainsi, même si ce travail est indéniablement centré sur l'ère contemporaine, il ne faudrait pas négliger 
l'importance des courants et des tendances poétiques qui ont caractérisé la fin du XIX ${ }^{\text {ème }}$ et le début du XX ${ }^{\text {ème }}$ siècle, comme le surréalisme ou les avantgardes entre autres, parce qu'ils ont bouleversé le paysage poétique à jamais, en introduisant des nouveautés et des révolutions esthétiques, thématiques et formelles qui ont marqué le pas du devenir poétique jusqu'à nos jours. Après le romantisme, la fin du XIX ${ }^{\text {ème }}$ siècle a vu naître des figures comme Baudelaire, qui introduira la ville moderne et la laideur en poésie, ainsi que des nouveautés formelles comme le poème en prose ; Rimbaud, qui a préconisé « le dérèglement de tous les sens » (Rimbaud, 1871) et a remis en question l'emploi du langage à travers un esprit de subversion ; Lautréamont, qui, avec ses Chants de Maldoror (1869), a poussé à l'extrême cet esprit subversif en attaquant la moralité à travers l'apologie du mal ; ou Mallarmé, qui a revendiqué que « la poésie est un moyen de connaissance qui mène à l'absolu » (Leuwers 2009 : 79).

Le $\mathrm{XX}^{\text {ème }}$ siècle commence avec des poètes comme Paul Valéry, considéré l'héritier de Mallarmé, et qui a prôné le travail de la langue en refusant l'idée de l'inspiration poétique, ou Guillaume Apollinaire qui, avec sa conférence sur " l'Esprit Nouveau » a marqué la tendance des « jeunes créateurs [qui] ont un appétit de nouveauté et ne croient plus aux valeurs esthétiques du siècle passé » (Leuwers 2009 : 95). En même temps, les manifestes commencent à proliférer, en créant de nombreux mouvements d'avant-garde comme le surréalisme, qui marqueront le pas du devenir littéraire et poétique.

Les années 1950 se caractérisent par la fin du surréalisme et de la poésie dite « engagée », notamment associée aux mouvements de la Résistance qui ont surgi lors de la deuxième guerre mondiale. Dans celle qui est souvent connue comme l' " ère du soupçon " - en faisant allusion au titre de l'essai de Nathalie Sarraute paru en 1956 et dans lequel elle jetait les bases du « Nouveau Roman » en s'attaquant au roman traditionnel - des poètes comme Yves Bonnefoy, André du Bouchet, Philippe Jaccottet, Jacques Dupin ou Lorand Gaspar ont constitué le devant de la scène poétique. Il ne faut pas oublier que les années 1950 ont été marquées par la hantise de la seconde guerre mondiale, qui a poussé les poètes à se demander si la poésie ou, si l'écriture en général, était encore possible après une expérience aussi terrible que celle des camps de concentration et de l'holocauste nazi. D'après la célèbre formule du philosophe Allemand Théodor Adorno : "Écrire un poème après Auschwitz est barbare » (2003 : 26).

Dans les décennies qui ont suivi, la poésie française s'est notamment engagée dans une démarche expérimentale qui a dominé le paysage poétique 
jusqu'au début des années 1980. La figure de Jean-Claude Pinson pourrait être située dans une lignée poétique qui semble s’être développée en France à partir notamment des années 1980, et qui a tenté de récupérer un certain lyrisme poétique qui avait été négligé pendant les années 1960-1970 et les démarches poétiques expérimentales. C’est le cas de la revue Tel Quel, Change ou TXT, et de la naissance de la poésie sonore, dont Bernard Heidsieck et Henri Chopin ont été les principaux représentants, et qui continue de se développer aujourd'hui avec des poètes comme Christophe Tarkos, Charles Pennequin ou Nathalie Quintane, qui ont également participé à la revue Java en 1989 et qui a beaucoup exploré la « poésie-action » entre autres. Un autre représentant de ces tendances formalistes et expérimentales, c'est le collectif éditorial Orange Export Ltd. d’Emmanuel Hocquard, créateur de la dénomination de la "modernité négative ». Pour ce qui est de la poésie " minimaliste » ou " blanche », il faut parler d'André du Bouchet, qui a conduit la poésie à l'épuration des formes. Une autre tendance caractérisée par l'innovation formelle, c'est le littéralisme de Jean-Marie Gleize, qui a revendiqué l'abandon de la métaphore et des images. Il faudrait également parler de la parution, en 1988, de l'Art Poétic' d'Olivier Cadiot, qui met en place une poésie fragmentée dans laquelle il s'adonne à la reproduction textuelle. Finalement, nous mentionnerons la Revue de littérature générale, fondée par Olivier Cadiot et Pierre Alferi, dont le premier numéro parut en 1995, sous le titre de La mécanique lyrique, et dans lequel le ton anti-lyrique et favorable aux expérimentations formelles fut instauré.

À partir des années 1990 les démarches déconstructivistes et expérimentales continuent de se développer, avec des projets comme la revue Nioques. La poésie s'est rapprochée des arts plastiques, de la photographie et du cinéma. Par exemple, en l'an 2000, un groupe constitué de peintres, poètes, photographes, sculpteurs, etc. s'est rassemblé autour d'Éric Sivry et de la revue Intuitions, dans le but d'explorer la composante intuitive de tout acte de création artistique. Néanmoins, parallèlement à ce développement de tendances déconstructivistes et expérimentales, une poésie plus attentive à la voix, au chant et au rythme continue également de prospérer, avec des poètes proches de démarches plus lyriques de la poésie.

En ce qui concerne la fin du $\mathrm{XX}^{\text {ème }}$ siècle, le poète et critique Jean-Michel Espitallier fait un diagnostic des dernières années du siècle, dans lesquelles la poésie semble vouloir continuer d'explorer de nouveaux chemins, mais aussi reprendre d'anciennes questions et tenter de fournir des réponses inédites : 
La dernière décennie du $\mathrm{XX}^{\text {ème }}$ siècle a vu la poésie française se renouveler en profondeur, ouvrir des voies inexplorées, reposer en termes neufs les questions de son statut, de ses limites et de ses formes. C'était inespéré. Dans un contexte un peu morose qui n'en finissait pas de proclamer la fin des avant-gardes sans proposer beaucoup mieux qu'un vague lifting de la vieillerie poétique, on aurait dit que la machine, enfin, se remettait en marche (2011: 15).

Il ne faut pas oublier que, depuis les dernières années du XX ${ }^{\text {ème }}$ siècle et, notamment dans ces premières années du XXI⿳亠े⿵冂丶 ${ }^{\text {me }}$ siècle, la poésie a également été marquée par le développement incessant de l’univers numérique et aussi par l'arrivée d'Internet.

Face à ce panorama dans lequel nous avons annoncé les principaux protagonistes de l'expérimentation formelle contemporaine, Jean-Claude Pinson se situerait dans une position assez éloignée de ces innovations formelles et expérimentales, et plutôt proche d'une poésie lyrique. C'est ce que nous constatons dans ces propos de Jean-Michel Maulpoix, probablement l'un des auteurs les plus engagés dans la défense du lyrisme contemporain - ou plutôt, du « Nouveau Lyrisme ». Par exemple, dans l'essai La poésie comme l'amour (1998), Maulpoix résume cette opposition entre lyrisme et littéralisme qui nous aide à comprendre la place que des poètes contemporains comme Pinson occupent par rapport à l'expérimentation formelle :

Entre 1960 et 1970, l’on a vu en effet se développer, dans la proximité des sciences humaines, des travaux poétiques concentrant l'essentiel de leur attention sur la production de l'objet littéraire, sa littérarité ou sa littéralité. [...] Dans cette perspective, fut largement remis en cause le statut « lyrique » du texte poétique, tel qu’il donne à imaginer une continuité idéale entre les mots et les choses. [...] Si le lyrisme réapparaît, c'est donc au moment où reflue cette scientificité et où s'engage la « critique de la critique ». Le lyrisme revalorise alors la notion « d'expérience poétique » contre l'affirmation telquelienne de Denis Roche selon laquelle « toute révolution ne peut être que grammaticale ou syntaxique ». Ce retour du/au lyrisme se situe pour une part dans un déplacement de l'attention de la page blanche [...] vers le monde. Le lyrisme ne peut prendre son essor qu'en se dégageant des préalables théoriques tels qu'ils tendent à isoler l'écriture en la détachant des autres activités humaines. Il implique l'affirmation renouvelée d'une interdépendance étroite entre l'écriture et la vie (1998: 120-122).

Les années 1980 ont ainsi été caractérisées par la volonté de quelques poètes de revisiter et de réinterpréter le penchant lyrique de la poésie. Ces poètes ont 
voulu rompre avec les démarches expérimentales des décennies précédentes, qui se seraient limitées à demeurer dans la dimension de l'expérimentation du langage et des mots et auraient provoqué l'appauvrissement et la stérilisation de la poésie. C'est pourquoi, à partir des années 1980 notamment, certains poètes ont fait l'effort d'ouvrir une nouvelle dimension poétique où des notions lyriques de la poésie telles que la musicalité des mots et du langage, la présence de l'image, l'expression du " moi » poétique et même de l'émotion, seraient possibles, tout en évitant de reprendre la voie de l'épanchement lyrique du romantisme traditionnel. Ces poètes ont fréquemment été désignés comme des " nouveaux lyriques », une dénomination qui souligne leur volonté de laisser de côté l'esprit intellectualiste et abscons qui avait marqué la poésie expérimentale de la période précédente et ils se sont regroupés autour de la notion de " Nouveau Lyrisme » et de « Lyrisme Critique », deux concepts sur lesquels le poète et essayiste JeanMichel Maulpoix a longuement théorisé.

En effet, il semblerait que Pinson, ainsi que d'autres poètes contemporains comme James Sacré, avec des recueils tels que Si peu de terre, tout (2000), Une petite fille silencieuse (2001), Le poème n'y a vu que des mots (2007), America solitudes (2010), ou Un effacement continué ? (2016), Antoine Emaz, avec des recueils comme Ras (2001), K.O. (2004), Caisse claire (2007), Cambouis (2009), De Peu (2014) ou Limite (2016), Yves Leclair, avec des ouvrages tels que L'or du commun (1993), Manuel de contemplation en montagne (2005), Orient intime (2010), ou Cours s'il pleut (2014), ou encore Guy Goffette, avec des recueils comme Éloge pour une cuisine de province (1988), La vie promise (1991), Un manteau de fortune (2001), ou Petits riens pour jours absolus (2016), entre autres, se permet de déclencher un certain lyrisme poétique sur la page, mais qui demeure en contact avec la réalité et qui ne vise ni l'évasion ni l'élévation. En plus, le rapport que Pinson entretient avec la partie musicale de la poésie est très fort, parce qu'il considère que la sonorité des mots se trouve à l'origine des langues, qu'elle en constitue l'essence. La poésie représente une sorte de hautparleur du monde pour lui, dans la mesure où elle veut faire résonner les bruits du monde en elle, en se servant de la composante musicale et sonore des mots qui sont chargés de transmettre le bruit du monde au sein du poème.

Cette démarche poétique qui caractérise l'œuvre de Pinson en demeurant proche d'un lyrisme qui lui permet de renforcer le lien entre le fait poétique et la vie, est celle que nous aimerions analyser dans les pages qui suivent, parce qu'il nous semble qu'elle est à la base du travail de ce poète contemporain. Pour ce faire, nous tenterons d'explorer les concepts de " poéthique » et de 
« poétariat », souvent énoncés par Pinson, dans l'espoir de saisir l'essence du travail que ce poète réalise toujours à cheval entre la poésie et la philosophie. De même, nous étudierons quelques extraits de ses recueils poétiques, qui nous aideront sans doute à illustrer ces notions car, il ne faut pas oublier que l'œuvre de Pinson relève toujours d'une double démarche philosophique et poétique indissociable, qui fait que son œuvre se rapproche de ce que Pinson lui-même, dans son essai Habiter en poète (1995), qualifie de " poésie pensante ", en citant la démarche de poètes comme Yves Bonnefoy, Philippe Jaccottet ou Michel Deguy, qui ont également exploré les zones de partage entre la philosophie et la poésie :

On constatera d'abord qu'existent aujourd'hui, quelque discrète que soit leur audience, des œuvres non négligeables, dont la teneur quasi philosophique est peu discutable : celles d’Yves Bonnefoy, de Philippe Jaccottet ou de Michel Deguy par exemple. La poésie donne à penser jusque dans les diapositifs où elle travaille à la défection du sens. Il fait prendre au sérieux ce qu’une poésie peut donner à penser à sa façon en apparence la moins « pensante », la plus légère. Car « pensante » ne signifie pas « pesante » (1995: 55)

\section{2. "Poéthique "}

Dans sa quête d'une poésie toujours liée à la philosophie, Jean-Claude Pinson a souvent voulu analyser le versant existentiel et anthropologique touché par la poésie, c'est-à-dire la dimension du vécu que l'acte poétique parvient à atteindre au-delà de son ambition esthétique : «Quelle est sa façon singulière, spécifique, de suggérer des formes de vie expérimentables hic et nunc et capables de donner une autre qualité (une autre intensité, une autre vitesse) à l'existence ? » (2013 : 37).

C'est dans cette tentative que Pinson fait souvent allusion au terme " poéthique », avec lequel il souhaiterait aborder le lien que la poésie s'efforce de maintenir avec le vécu. La " poéthique » constituerait donc l'extension de la poésie dans la vie de chacun. D’après Pinson, la poésie élargit son lien avec la vie et c'est la notion de " poéthique » qui permet d'intensifier cette combinaison entre ce qui est dit et ce qui est vécu. Ainsi, grâce à cette notion de " poéthique ", nous arrivons à mieux comprendre le choix de Pinson pour une poésie plutôt lyrique, c'est-à-dire une poésie qui garde le contact avec le vécu, contrairement à une poésie formelle qui demeure dans la dimension du 
langage comme expérimentation, ainsi que la chercheuse Laure Michel le montre dans son article " Crise de la poésie ? Le poétariat selon Jean-Claude Pinson » (2012) : « La « poéthique » signe le refus d’une poésie enclose dans les limites du langage » (2012 : 250). Voilà pourquoi Pinson insiste souvent sur le lien entre poésie et vécu que le terme " poéthique » permet de désigner :

\begin{abstract}
Telle est la question que j’appelle « poéthique », celle qui considère que la poésie n’est pas que la poésie qui s'écrit (ou se " performe »), mais aussi ce qui d'elle existe et vaut hors d'elle-même. Car si elle n'est pas seule, c'est d'abord parce que le poème est solidaire, comme dit Thoreau, de ce qui tente, au verso de la feuille, de s'écrire dans la vie (Pinson 2008a : 53).
\end{abstract}

De même, la notion de " poéthique " permet à Pinson de consacrer une place sociale à la poésie et de lui faire jouer un rôle politique, en tant que vecteur entre le fait poétique et le vécu, car dans la mesure où « l'écriture a la capacité de contribuer à la constitution d'une "éthique de soi", elle peut transformer un mode d'existence » (2012 : 251). D’après Pinson, le poète serait donc un « accentueur d'existence », puisque la distance que la poésie garde avec le réel lui permet d'aborder celui-ci d'une manière particulière et plus intense. Cette composante politique et sociale dont témoigne la notion de " poéthique » est résumée par Pinson dans un extrait de son essai À Piatigorsk sur la poésie (2008a), où il parle de la démocratisation de l'art qui a lieu dans le contexte contemporain, et qui conduit sans doute la poésie à développer sa teneur politique :

Mettre l'accent sur la portée « éthopoiétique » de la poésie n'est pas sans incidence politique. Car ce qui est par là retrouvé, c’est le devenir " démocratique " d'une poésie (d'une littérature) de plus en plus écrite par tous depuis qu’elle est entrée, avec les autres arts, dans l'âge de la multitude artistique (2008a : 63).

Toujours dans ce même essai, nous retrouvons les propos de Pinson, qui souligne que la composante éthique de la poésie recueillie dans le mot « poéthique », constitue l'une des principales ambitions de tout acte poétique. Donc, nous pourrions affirmer que pour Pinson, la " poéthique » représente l'espace où confluent la poésie et la philosophie avec l'intention de parvenir à la part de vécu que tout acte poétique devrait comprendre : « La finalité de l'acte d'écrire [...] n'est pas d'abord dans l'attente d'un retour esthétique, mais éthique, existentiel » (2008a : 58), d’où le terme " poéthique ». 
Après avoir révisé les propos plutôt théoriques que Pinson a développés dans ses essais sur le concept de "poéthique ", nous nous arrêterons sur son travail poétique, ce qui nous permettra de constater que son dessein d'établir et de maintenir un lien entre la poésie et le vécu, constituent non seulement un souci théorique ou philosophique pour lui, mais aussi le ferment de son travail avec les mots.

Tout d'abord, il faut dire que Pinson n'est pas le seul à mettre en relief ce rapport entre le fait poétique et la vie dans le contexte poétique contemporain. En fait, nombreux sont les auteurs actuels qui suivent cette même démarche. Par exemple, dans cet extrait de l'essai sur la poésie contemporaine La poésie sauvera le monde (2015), le poète et essayiste Jean-Pierre Siméon souligne l'importance de ce lien entre la poésie et le vécu :

La poésie ne s’en tient jamais à la description-narration des faits et mécanismes de surface (le surf sur le réel qui devient à présent le mode obligé de la saisie du monde), cette pseudo-réalité qui n'est du réel que son fantasme, son reflet immobile, un miroir aux alouettes. Le poème ne cherche pas à contenir le réel, n’est pas cette contention du réel qui vise à l'inventorier en représentations stables et repérables, en conventions donc. Il tente d'en percevoir l'extension infinie dans la résonance qu'il a dans la conscience. Le réel de surface, tangible et visible, est inventoriable (le récit dresse cet inventaire), le réel dont nous entretient la poésie, non pas celui qui se décrit mais celui qui se vit, est comme la vie qui l'agit, infini (2015: 40).

Cette intention de « nouer le vivre et l'écrire » est également mise en relief dans l'essai que Pinson a écrit en 1995, intitulé Habiter en poète, dans lequel il affirme qu'il s'agit là d'une inquiétude qui caractérise le paysage poétique actuel :

La donne nouvelle qui caractérise l'actuel paysage de la poésie présente le grand intérêt de porter au premier plan la question de l'habitation poétique. [...] Mais on est aujourd'hui attentif, peut-être plus qu'hier, à nouer le vivre et l'écrire pour faire que la vie soit vraiment « habitante ». [...] La poésie contemporaine, comme aventure au croisement du langage et de l'existence, demeure un objet de pensée digne d'intérêt (1995 : 16).

En ce qui concerne son travail poétique proprement dit, l'une des pistes qui nous indique le goût de Pinson pour ce contact entre la poésie et la réalité ou la vie, apparaît dans une citation qui ouvre son recueil J'habite ici (1991). Ce 
recueil s'initie avec une citation de Goethe qui marque le ton de ce qui va suivre, et dans laquelle Goethe revendique « une poésie de circonstance », une poésie qui découle de la réalité : « Le monde est si grand, si riche, et la vie offre un spectacle si divers que les sujets de poésie ne feront jamais défaut. Mais il est nécessaire que ce soient toujours des poésies de circonstance, autrement dit il faut que la réalité fournisse l’occasion et la matière » (1991 : 7).

Dans cet autre exemple du recueil Free jazz (2004), Pinson joue avec la double démarche menée par les mots entre la dimension de l'écriture et celle de la réalité, ce qu’il représente à travers un petit jeu de mots avec les termes " page » et « plage ». L'auteur semble obsédé par le fait de faire que les mots entrent en contact avec la réalité, il ne veut pas s’éloigner du vécu, et cette comparaison de la page avec la plage lui permet de maintenir le poème en contact avec le réel :

L'éternel problème de faire vivre au grand air des mots qui restent obstinément scotchés sur la page (je les engueule comme des gosses visés devant la télé : allez donc jouer sur la plage !)

me répondent que pas question pour eux de jouer le jeu du livre de plage (2004 : 34).

Ainsi, le poète compare ses promenades sur la plage avec ses « promenades » sur la page, profitant de la grande ressemblance des deux mots, " page » et " plage », qui devient finalement un prétexte pour rapprocher au maximum la dimension poétique de la réalité et du vécu : « $\mathrm{P}(\mathrm{l})$ age : la page et la plage. Chaque jour je va-et-viens de l'une à l'autre, comme entre deux épouses. Installé, quoique sédentaire (très exactement : fonctionnaire à la plage, occupé de livres et marées, de pensées et nuages, de marches énergumènes) » (2004 : 23).

Finalement, le poète reste divisé entre ces deux espaces, la page et la plage, c’est-à-dire la dimension poétique et la réalité, cette double présence à laquelle aspire Pinson dans sa poésie. Autrement dit, le poète ne fait pas le choix entre la « page » ou la « plage », il n’exclut ni la vie ni les mots de son travail poétique, parce qu’il considère que la véritable poésie, celle qu’il voudrait atteindre, est toujours concernée par les deux, et c’est précisément quand le poète parvient à partager et à mettre en rapport ces deux territoires, celui des mots et celui de l'existence, que le poème surgit véritablement : " donc je danse d'un pied sur l'autre, tantôt à la plage, tantôt à la page » (2004 : 29).

Cette volonté de faire que le vécu fasse partie du poème, attribuerait même au poète la faculté de devenir un « soignant », de soulager d’une certaine manière 
les fluctuations auxquelles on est soumis au cours de l'existence, tel que cet extrait du recueil Drapeau rouge (2008b) nous le fait constater :

Le poète n'a plus qu'à se faire acupuncteur. Être un soignant (de soi, des vivants et des morts, du monde), qui cherche avec des phrases les bons méridiens, les bonnes déclinaisons d'atomes. Ou bien un secouriste pratiquant le bouche-à-bouche, un réanimateur sachant souffler sur les plus fragiles braises de vie. Un chamane [...] On ne peut compter, pour guérir, que sur l'écriture et la page (2008b : 108-109).

La notion de " poéthique » constitue donc pour Pinson l'outil nécessaire pour produire et étudier les textes poétiques non seulement comme des objets esthétiques, mais comme des modèles d'existence possibles, et par conséquent, de devenir source de métamorphose de cette même existence : « La poésie (et plus largement la littérature) ne vaut pas seulement per se, comme simple production de textes et de livres, mais comme moyen, en vue d'une existence qu'elle aiderait à « changer » » (2008a : 60).

\title{
3. « Poétariat"
}

Lorsque Pinson tente de répondre à la question de la continuité et de la survie de la poésie dans le contexte contemporain, il commence par avouer sa place de minorité et de marginalité, ainsi que nous le voyons dans le recueil J'habite ici (1991), où le poète semble être conscient de la minorité que représentent les lecteurs et les amateurs de poésie de nos jours :

\author{
Il y a juste ici deux ou trois librairies \\ et il faut bien chercher \\ pour dénicher quand elles arrivent \\ les nouveautés de poésie \\ mais après tout combien d'égarés sommes-nous \\ qui à tout prix avons besoin de tels produits ? (1991 : 51).
}

Ces considérations sur l'état actuel et sur le sort de la poésie de nos jours sont repris dans le recueil Fado (avec flocons et fantômes) (2001), où, en faisant allusion aux propos de Maurice Blanchot, le poète affirme que la poésie tend à disparaître, tel que Blanchot l'avait prévu : « décidément, Blanchot n’a pas tort : la poésie va vers son extinction. Sa disparition. / Retourne, peut-être, à son 
origine nocturne. Au néant. / Ah ! la fascination du silence, les prestiges de la ténèbre et du secret » (2001: 85).

Dans un article intitulé « Poésie : un regain ? " (2017), publié dans la revue Carnets en 2017, Pinson résume la position minoritaire de la poésie, et, en faisant allusion aussi bien au plan économique que symbolique, il fait référence aux propos de Baudelaire quant à la perte de l’auréole de la poésie :

La poésie, en France, n’est-elle pas au plus bas ? Tout semble du moins l’indiquer. Au plan économique, sa place dans l'édition est de plus en plus réduite, écrasée qu'elle est, plus que jamais, par le roman. Absente de plus en plus des librairies, elle n'a plus, ou presque, de lectorat. [...] Au plan symbolique même, son prestige d'autrefois semble n'être plus qu'un souvenir. Plus d’instance supérieure pour légitimer sa parole (divinité, Muse...) ; plus de fonction définie (la poésie institutrice de l'humanité, conférant une valeur mythique et un rythme sacré à la geste collective...). Dans la ville moderne, le poète, comme déjà le constatait Baudelaire sur le ton de la dérision désabusée, a perdu son auréole (2017 : 1).

Néanmoins, malgré cette prise de conscience sur la situation de minorité de la poésie dans l'actualité, Pinson avoue qu’elle continue d’exister, parce que, malgré la perte d’autorité et de légitimité apparente du poète, un désir de chant dans la langue persiste toujours. C'est ce que nous constatons dans ce passage de l'essai Sentimentale et naïve (2002) : « C’est un fait, ténu peut-être, mais têtu : il y a de la poésie, toujours, encore, de nouveau. De la poésie plurielle, foisonnante et insaisissable, provocante ou discrète, insipide ou excitante, hérissée ou limpide, voyante ou peu visible, endogène ou exogène, etc. » (2002 : 11).

D’après Pinson, cette position de minorité et de marginalité de la poésie est due, entre autres, au changement d'époque qui nous a fait passer de l'âge industriel à l’âge post-industriel, une évolution qui a provoqué la fin de la classe ouvrière et le surgissement du consommateur de masse, que Pinson désigne souvent avec le terme de « Homo œconomicus » (2012 : 111). D’après Pinson, la société actuelle se caractérise par l'émergence de ce qu’il qualifie de « capitalisme culturel » (2012 :110), capable d'exercer un contrôle social sur le consommateur de masse soumis aux exigences et aux modèles instaurés par la surmédiatisation, le marketing et les technologies de la communication.

Mais, Pinson n’est pas le seul à avoir mis l'accent sur le devenir de la société actuelle qui bascule vers ce modèle post-industriel. Nous pouvons citer les propos de l'économiste français Daniel Cohen qui, dans son œuvre Homo 
Economicus, prophète (égaré) des temps nouveaux (2006), décrit ainsi cet « homo economicus » souvent cité par Pinson lui-même :

Le paradoxe central de l'époque est toutefois le suivant. L'économie est sommée de prendre en charge la direction du monde à un moment où les besoins sociaux migrent vers des secteurs qui peinent à s'inscrire dans la logique marchande. La santé, l'éducation, la recherche scientifique, le monde d'Internet forment le cœur de la société post-industrielle. Aucun n'entre dans le moule économique traditionnel. Alors que la créativité humaine est plus élevée que jamais, Homo economicus s’impose comme un triste prophète, un rabat-joie des temps nouveaux (2006: 7-8).

Très critiques sont également les affirmations de l'écrivain et essayiste JeanMichel Espitallier qui, dans son essai sur la poésie contemporaine Caisse à outils : un panorama de la poésie française aujourd'hui (2014), nous montre un regard très réprobateur des caractéristiques propres à la société moderne, qu’il qualifie avec des termes aussi durs que " sédentarité répétitive » ou " surface plate » et, en même temps, il se demande si le poète a encore la possibilité de trouver sa place au sein de cette société qu'il condamne durement :

La société moderne, condamnée à ne plus être qu’une reproduction, prise en charge par la fiction, déréalisée, nous a privés de toute possibilité d’expérience en nous fixant dans une sédentarité répétitive où le travail est devenu un emploi, l’individu une ménagère de moins de 50 ans, le voyage un congé, la vieillesse une retraite. Dans ces conditions, peut-on habiter le monde en poète si le monde n'est plus qu'une surface plate, un miroir vide, une fiction ? (2014: 77).

Face à ce développement social qui nous conduit vers un modèle postindustriel fortement marqué par la consommation de masse et le contrôle social exercé par les médias, Jean-Claude Pinson insiste notamment sur le surgissement de nouvelles formes de travaux et de travailleurs : « le phénomène majeur suscité par les nouvelles technologies est bien l'apparition de formes nouvelles de travail » (2012 : 105). En plus, lorsque Pinson évoque l'émergence de ces nouveaux types de travailleurs qui ne sont plus dans la lignée du travail industriel, il cite les propos du philosophe Antonio Negri, tirés de son essai Traversées de l'Empire (2011), où il utilise la notion de " travail immatériel » pour désigner le travail accompli par cet « homo œconomicus » post-industriel : 
Les transformations du capitalisme, l'importance prise par les technologies de l'information et le travail immatériel ont en effet mis fin dans nos contrées à l'hégémonie, économique autant que symbolique, du travail industriel et de la classe ouvrière. " Dans la dernière décennie du XX ${ }^{\text {ème }}$ siècle, écrit Antonio Negri, le travail industriel a perdu son hégémonie, et c'est le "travail immatériel” qui a émergé à sa place, c'est-à-dire un travail qui crée des produits immatériels : le savoir, l'information, la communication, les relations linguistiques ou émotives » (2012:105).

En plus, Pinson souligne également que ce nouveau modèle social caractérisé par le contrôle du capitalisme culturel, a également conduit à une démocratisation de l'activité artistique. En rapport avec ceci, le chercheur Laurent Zimmermann a publié un article intitulé « Jean-Claude Pinson, Hobby et Dandy. Sur l'art dans son rapport à la société » (2004), dans la revue Littérature :

Que la poésie, ou la peinture, ou la musique, etc., soient faites « par tous » et pour tous, est d'une certaine façon ce qui ne cesse plus d'advenir dans les sociétés démocratiques. Dans un tel infléchissement du rôle de l'art se trouve le ferment de sa transformation en « hobby ", avec tous les dangers que cette transformation implique (2004 : 121).

Face à ce panorama de transformation sociale vers le modèle de la démocratisation de l' art et vers le modèle de l' " homo œconomicus », soumis à la logorrhée des médias télévisuels qui s'adressent à la multitude, et à la massification des mœurs et des pratiques culturelles provoquée par les nouvelles technologies médiatiques, Pinson revendique la notion de « poétariat » comme celle qui aurait la force de résister à cet aplatissement provoqué par le changement d'époque. Selon Pinson, le « poétariat » aspire donc à « inventer de formes de vie plus sobres, plus "soutenables" [...] de se sauver du désastre où conduit aujourd'hui le règne de l'Homo œconomicus et des catégories qui lui sont attachées » (2012: 114).

Néanmoins, Jean-Claude Pinson avoue qu'il n'est pas le premier à avoir utilisé ce terme de " poétariat ». Apparemment, un poète dadaïste appelé René Edme, l'aurait utilisé en 1920 dans un ouvrage intitulé Poétariat ou l'immorale vie de Safran Corday (1982), publié 60 ans après sa mort par Georges Schmits. Pour Pinson, le terme fait plutôt référence au surgissement d'une nouvelle époque marquée par la démocratisation de l'art, une époque où l'expression artistique a cessé d'appartenir exclusivement à quelques-uns, pour se transformer en ferment de la métamorphose de l'individu moderne, prêt à s'inscrire dans le monde à travers l'expression artistique : 
Si j'ai eu recours pour ma part à ce néologisme de « poétariat », c'est d'abord pour penser les évolutions propres de la poésie. Pour saisir notamment la transformation et la démultiplication de son « scriptorat » à la faveur des technologies numériques. [...] L' « objectivité » du mot, son poids de réalité, tient au fait que la notion (c'est du moins ce qui m’est apparu) aide à penser, bien au-delà de la seule poésie, la levée en masse, en régime démocratique, d’une armée toujours plus nombreuse d'artistes ou d'aspirants artistes. Au-delà, elle permet sans doute de capter quelque chose d'essentiel quant au changement d'époque qui nous affecte et qui voit surgir, bien au-delà du " poétariat » au sens restreint (celui des poètes friands de plaquettes ou de blogs), une multitude « artiste » hors des lieux balisés de l'art : tout un « poétariat » au sens très élargi, attaché à modeler et gouverner sa vie, à customiser au mieux son existence au moyen de tout le spectre des arts du quotidien (2012 : 109-110).

Il est donc manifeste que, d'après Pinson, la notion de « poétariat » implique une attitude de résistance au modèle politique et social préconisé par la société de consommation de masse actuelle et, par là même, contribue à concevoir d'une nouvelle manière le rapport que la poésie maintient avec la politique. C'est ce que la chercheuse Laure Michel affirme dans son article sur Jean-Claude Pinson :

Le poétariat désigne cette tendance massive de la multitude à l'invention de nouvelles formes de vies, de relations, d'affects et de subjectivités, qui résistent au biopouvoir. La notion a le mérite de mettre l'accent sur la dimension poïétique, créative, de cette résistance (2012 : 255).

C'est-à-dire en s’opposant au modèle de l' ' homo œconomicus », qui conçoit l'individu moderne comme un sujet soumis à l'utilitarisme et qui est jugé selon ses revenus, ses possessions et est encouragé à se plier aux intérêts économiques, Pinson présente le modèle de "l'homo poeticus ou artisticus », qui cherche un accomplissement plus complet de l'individu :

Le modèle aujourd'hui dominant, celui de l'Homo oeconimicus, pense l'individu comme astreint au principe utilitaire [...] Il l'évalue selon son revenu et l'invite à la possession de biens toujours plus nombreux, l'incite à privilégier l’intérêt à court terme, l'utilité comprise en un sens étroit. Il presse le système éducatif et le monde universitaire de s'adapter toujours davantage aux impératifs de l'économie et aux contraintes de la compétition internationale. Le modèle adverse, celui de l'Homo poeticus (ou artisticus) est en quête d'une existence plus accomplie, d'un meilleur épanouissement pour chacun de ses facultés diverses (2012:115). 
En ce qui concerne l'œuvre poétique de Pinson, il faut dire que ce changement d'époque apparaît souvent reflété dans ses poèmes, ainsi que nous le constatons dans son recueil Drapeau rouge (2008b), où il fait toute une réflexion sur la société actuelle et la rapidité qui la caractérise, à partir de la disparition de la voyelle « o » du mot « saxo ». À son époque, quand le poète était jeune, il affirme qu'on appelait cet instrument de musique qu'il évoque dans le poème « saxo » mais, maintenant, on l'appelle « sax » tout court, ce que le poète interprète comme un signe de changement d'époque, et comme la marque de la vitesse qui caractérise notre ère, et aussi notre écriture actuelle, plus penchée vers la vitesse et le raccourcissement que vers des fragments longs. Autrement dit, c'est à partir d'un petit changement dans un mot que l'auteur tire toutes ces conclusions sur la vitesse caractéristique de nos sociétés modernes, qui influence inévitablement la manière dont les poètes abordent le travail avec les mots, en les rendant plus favorables à des passages brefs et fragmentés, plutôt qu'en les renvoyant à des formules plus longues et étendues :

\begin{abstract}
[...] le mot sax(O).
Erreur ! Erreur d'époque ! nous a-t-on immédiatement fait savoir. Car on dit sax aujourd’hui, sans plus. Ce n'est plus dans le tempo, paraît-il, le O bleu de saxo. Trop daté, trop rétro, nous a fait remarquer Vico Sponni, un musico de nos amis

ça n’a l'air de rien, cette histoire de voyelle qui disparaît. Mais en fait, c'est tout un changement d'époque qui s'y manifeste. En gros : la terre ne tourne pas saxo mais sax/. Question de rythme : plus rapide aujourd'hui, plus saccadé, plus techno. Et question de phrasé : plutôt cut-up que longues périodes, plutôt sex/ qu’amour de loin, etc. (2008b : 78).
\end{abstract}

De même, ce changement d'époque dont Pinson parle aussi bien dans ses essais que dans ses recueils poétiques, implique souvent un changement dans la conception du langage et dans son traitement dans la vie courante et en poésie. D'après Pinson, aussi bien le poète que l'individu moderne sont soumis à l'aplatissement du langage ordinaire. Devant cette réalité, Pinson, ainsi que d'autres poètes contemporains, attribuent à la poésie et à l'emploi des mots qu'ils font au sein du poème la tâche de lutter d'une certaine manière contre ce processus de banalisation du langage. Cette volonté de lutter contre l'emploi banalisé et plat du langage fait donc partie des principales inquiétudes de la poésie contemporaine, ainsi que nous le montre l'essayiste et critique littéraire JeanMichel Espitallier dans son essai sur la poésie contemporaine Caisse à outils : un panorama de la poésie française aujourd'hui (2014), où il parle de la poésie 
comme de celle qui nous permettrait d'avoir une attitude d' « insoumission » pour lutter contre la banalisation et la dégradation à laquelle est soumise la langue dans les échanges quotidiens : "La poésie, toujours radicalement présente, s’affirme d'abord comme une insoumission contre [...] le temps de la consommation et la marchandisation des discours avachissant-avachis. Langue d'une langue qui aurait perdu sa langue » (2014: 25).

Pour sa part, Jean-Claude Pinson n’hésite pas à critiquer le langage médiatisé et mensonger face auquel il nous faut trouver une vitesse appropriée qui nous permette de sortir de ces emplois corrompus, et de récupérer la véritable essence. De cette manière, la revendication d'une vitesse qui nous permette d'envisager un emploi du langage éloigné de ce que Pinson lui-même qualifie de « modèles aliénants de la société ", implique également que nous ayons un rapport plus étroit et plus authentique avec nous-mêmes, avec notre vécu, ainsi que nous le constatons dans cet extrait d'un entretien fait au poète dans la revue de littérature contemporaine Le matricule des anges :

\footnotetext{
Il y a d'abord ceci qu'en tant qu'êtres de parole nous ne sommes pas au monde de manière muette, même si le monde, lui, est muet. Mais quels sont les langages à notre disposition aujourd'hui pour aller vers le monde : celui de la marchandise, de la publicité, etc. ? Il y a une dimension fallacieuse dans le langage imposé par la société, et c'est à chaque individu de gagner sa propre langue contre un tel langage. Au fond, la lutte de chaque homme consiste pour lui à trouver sa bonne vitesse, que la recherche de cette vitesse passe par la poésie, par la danse, ou même, pourquoi pas, le travail du bois, pour autant que ce sont là des manières de se réapproprier soi-même et d'échapper aux modèles aliénants de la société. Celui qui ne se fait pas lui-même est dans la défaite. Il renonce (Laugier 2002 : 44).
}

\section{Conclusion}

Après avoir parcouru les principaux essais théoriques et quelques recueils poétiques de Jean-Claude Pinson, nous sommes en état d'affirmer que les concepts de " poéthique » et de "poétariat » constituent l'une des bases de la conception poétique et du travail avec les mots de ce poète français contemporain. En plus, ces deux notions nous permettent d'explorer l'un des principaux enjeux de son œuvre poétique, à savoir, son rapport indéniable à la philosophie et sa volonté permanente de faire de l'acte poétique un outil de saisissement du vécu sur la page. 
La " poéthique » constituerait la dimension où la poésie et la philosophie se rejoignent pour atteindre la composante existentielle de la vie et, à ce propos, lorsque Pinson cite la notion de " poéthique », il fait souvent allusion aux propos de Nietzche qui, dans le Gai Savoir (1882) affirmait ceci à propos des artistes et de la nécessité d'aller au-delà de l'art pour atteindre ce lien privilégié avec la vie :

\footnotetext{
Aller au-delà, c'est considérer la possibilité de son extension dans la vie elle-même, selon une logique dont Nietzche formule ainsi le programme dans le Gai Savoir : «nous devons, écritil, apprendre des artistes, en étant pour le reste plus sages qu'eux. Car chez eux, cette force subtile qui leur est propre s'arrête d'ordinaire là où s'arrête l'art et où commence la vie ; mais nous, nous voulons être les poètes de notre vie ». Commence alors, par-delà la poésie, l'espace de ce que j’appelle la « poéthique ». Où la poésie, la littérature, considérée sous l’angle de la philosophie, tend à devenir elle-même une forme de philosophie (2008a : 34-45).
}

Pour ce qui est du " poétariat ", il s'agit d'un concept qui permet à Pinson d'attribuer des capacités artistiques et créatrices à l'individu moderne, qui devient de nos jours un " poète en puissance » (2008a) : « la question de la poésie [...] est indissociable de l'émergence de ce que je nomme le “poétariat”. L’individu contemporain est un "poète en puissance”" (2008a : 54-55).

De même, Pinson évoque souvent la perte de contact avec le réel que l'individu contemporain subit dans le contexte actuel, où tout devient de plus en plus intangible et où la fréquentation de l'expérience du réel devient de plus en plus insaisissable : « chute de l'expérience, perte de l'expérience vive des choses et du monde. Il ne reste que "le spectacle et la consommation" " (2008a : 97). Face à cette réalité, Pinson revendique la poésie comme le moyen de maintenir ou de récupérer ce contact avec le réel et, dans cette reprise de contact avec le réel, les notions de " poéthique » et de " poétariat " s'occupent de renforcer le lien entre la poésie et le vécu à travers une volonté de renouer avec un langage poétique qui permette d'établir un certain rapport sensible au monde.

Par contre, il faudrait rappeler que ce rapport que Pinson tente de maintenir dans ses poèmes avec la réalité et le vécu, faisant ainsi une poésie que nous pourrions qualifier de circonstance, n’est pas exclusif de son œuvre poétique, il constitue une caractéristique commune à toutes les écritures poétiques contemporaines, tel que le poète et essayiste Jean-Michel Maulpoix l'affirme dans son essai Pour un lyrisme critique (2009). D’après Maulpoix, c'est dans la manière dont chaque poète envisage ce rapport à la réalité de la vie courante et dans la manière dont il dit ce rapport sur la page, que réside la différence qui 
nous permet de distinguer des tendances et des orientations différentes au sein de la poésie contemporaine :

Sans doute est-ce du côté du rapport à la réalité qu'il faut chercher le commun dénominateur des écritures poétiques contemporaines. [...] Pour certains le réel est ce qui est impossible à dire, tandis que pour d'autres il est constamment approché à travers le quotidien, la circonstance, les objets et les événements ordinaires de la vie. [...] Qu'elles soient lyriques, littéralistes ou constructivistes, les écritures d'aujourd'hui prennent en compte la vie commune, afin de la dire au plus près, sans l'embellir ni la falsifier (2009 : 93-94).

\section{Références}

ADORnO, T. (2003). Prismes. Critique de la culture et de la société. Paris : Payot.

COHEN, D. (2006). Homo economicus : prophète (égaré) des temps nouveaux. Paris : Albin Michel.

DE CERTEAU, M. (2014). L'invention du quotidien : 1. Arts de faire. Paris : Folio.

EMAZ, A. (2001). Ras. Saint-Benoît-du-Sault : Tarabuste.

EMAZ, A. (2004). K.O. Paris : Inventaire-invention.

EMAZ, A. (2007). Caisse claire : Poèmes 1990-1997. Paris : Points.

EMAZ, A. (2009). Cambouis. Paris : Du Seuil.

EMAZ, A. (2014). De Peu. Saint-Benoît-sur-Sault : Tarabuste.

EMAZ, A. (2016). Limite. Saint-Benoît-sur-Sault : Tarabuste.

ESPITALLIER, J-M. (2011). Pièces détachées : une anthologie de la poésie française d'aujourd'hui. Paris : Pocket.

ESPITALLIER, J-M. (2014). Caisse à outils : un panorama de la poésie française aujourd'hui. Paris : Pocket.

GOFFETTE, G. (1988). Éloge pour une cuisine de province. Paris : Gallimard.

GOFFETTE, G. (1991). La vie promise. Paris : Gallimard.

GOFFETTE, G. (2001). Un manteau de fortune. Paris : Gallimard.

GOFFETTE, G. (2016). Petits riens pour jours absolus. Paris : Gallimard.

LAUGIER, E. (2002). "La pensée du dehors ». Le matricule des anges 41 : 44-45.

LECLAIR, Y. (1993). L'or du commun. Paris : Mercure de France.

LECLAIR, Y. (2005). Manuel de contemplation en montagne. Paris : La Table ronde. 
LECLAIR, Y. (2010). Orient intime. Paris : Gallimard l'arpenteur.

LECLAIR, Y. (2014). Cours s'il pleut. Paris : Gallimard.

LEUWERS, D. (2009). Poètes français des XIX̀̀me et XX $X^{\text {ème }}$ siècles. Paris : Librairie Générale Française.

MAULPOIX, J-M. (1998). La poésie comme l'amour. Paris : Mercure de France.

MAULPOIX, J-M. (2009). Pour un lyrisme critique. Paris : José Corti.

MICHEL, L. (2010). « Crise de la poésie ? Le poétariat selon Jean-Claude Pinson ». Les Temps Modernes 657 : 247-259.

NEGRI, A. (2011). Traversées de l'Empire. Paris: L’Herne.

PINSON, J-C. (1991). J'habite ici. Seyssel : Champs Vallon.

PINSON, J-C. (1993). Laïus au bord de l'eau. Seyssel : Champ Vallon.

PINSON, J-C. (1995). Habiter en poète : Essais sur la poésie contemporaine. Seyssel : Champ Vallon.

PINSON, J-C. (1999). À quoi bon la poésie aujourd'hui ? Nantes : Éditions pleins feux.

PINSON, J-C. (2001). Fado (avec flocons et fantômes). Seyssel : Champ Vallon. PINSON, J-C. (2002). Sentimentale et naïve : Nouveaux essais sur la poésie contemporaine. Seyssel : Champ Vallon.

PINSON, J-C. (2004). Free Jazz. Nantes : Joca Seria.

PINSON, J-C. (2008a). À Piatigorsk sur la poésie. Nantes : Cécile Defaut.

PINSON, J-C. (2008b). Drapeau rouge. Seyssel : Champ Vallon.

PINSON, J-C. (2012). « Du « poétariat » comme démenti au populisme ». Cités $49: 97-117$.

PINSON, J-C. (2013). Poéthique : une autothéorie. Seyssel : Champ Vallon.

PINSON, J-C. (2016). Alphabet cyrillique. Seyssel : Champ Vallon.

PINSON, J-C. (2017). " Poésie : un regain ? ». Carnets deuxième série 9 : $1-2$.

PINSON, J-C. (juin 1998). « Poésie pour « un peuple qui manque » ». Littérature $110: 34-35$.

PINSON, J-C. site officiel : https://www.jeanclaudepinson.fr/ (Dernier accès le 13 octobre 2018).

RIMBAUD, A., Lettre du voyant. 15 mai $1871<$ https://fr.wikisource.org/ wiki/Lettre_de_Rimbaud_\%C3\%A0_Paul_Demeny_-_15_mai_1871> (Dernier accès le 26 décembre 2016).

SACRÉ, J. (2000). Si peu de terre, tout. Chaillé-sous-les-Ormeaux : Le dé bleu. SACRÉ, J. (2001). Une petite fille silencieuse. Marseille : André Dimanche Éditeur. 
SACRÉ, J. (2007). Le poème n’y a vu que des mots. Chaillé-sous-les-Ormeaux : L'idée bleue.

SACRÉ, J. (2010). America solitudes. Marseille : André Dimanche Éditeur.

SACRÉ, J. (2016). Un effacement continué ? Nancy : La dragonne.

SIMÉON, J-P. (2015). La poésie sauvera le monde : essai. Paris : Le passeur.

ZIMMERMANN, L. (2004). « Jean-Claude Pinson, Hobby et Dandy. Sur l'art dans son rapport à la société ». Littérature 135 : 121-122. 